\title{
Weniger Hypoglykämie mit Flash-Glukose-Messung
}

Fragestellung: Kann die sensorbasierte Flash-Glukose-Messung (Free style libre) die Hypogykämierate im Vergleich zur herkömmlichen Glukoseselbstmessung (SMBG) reduzieren?

Hintergrund: Die engmaschige Kontrolle von Blutglukose bei Typ-1-Diabetes verzögert das Auftreten von makro- und mikrovaskulären diabetischen Komplikationen. Um Hypoglykämien zu vermeiden, müssen die Glukosewerte besonders eng überwacht werden.

Patienten und Methoden: In dieser multizentrischen, prospektiven, offenen, randomisierten Studie wurden Patienten mit gut eingestelltem Typ-1-Diabetes $\left(\mathrm{HbA}_{1 \mathrm{c}}<58 \mathrm{mmol} / \mathrm{l}\right.$ [7,5\%]) aus 23 europäischen Diabeteszentren eingeschlossen. Nachdem alle Teilnehmer 2 Wochen verblindet einen Sensor getragen hatten, wurden die Patienten, bei denen es Ergebnisse bei mindestens $50 \%$ gab, randomisiert in zwei Studienarme im Verhältnis 1:1. Die Interventionsgruppe erhielt das sensorbasierte Glukosemonitoring (Free Style libre), die Kontrollgruppe führte Blutzuckerselbstmessung mittels Teststreifen durch. Die Randomisierung wurde mit einer zentral gesteuerten Methode durchgeführt (biased-coin minimisation), jeweils abhängig vom Studienzentrum und Art der Insulingabe. Weder die Patienten, noch die Untersucher, noch das Studienpersonal wurden verblindet.

\section{Originalie}

Bolinder J, Antuna R, GeelhoedDuijvestijn P et al. Novel glucose-sensing technology and hypoglycaemia in type 1 diabetes: a multicentre, non-masked, randomised controlled trial. Lancet. 2016 Nov5;388(10057):2254-63. Der primäre Endpunkt war die Veränderung der Zeit-

Ergebnisse: Zwischen dem 4. September 2014 und 12. Februar 2015 wurden 328 Teilnehmer rekrutiert. Nach dem Screening und der Baseline-Phase wurden 120 Teilnehmer randomisiert der Interventionsgruppe und 121 Personen der Kontrollgruppe zugewiesen. Die Endpunkte wurden bei 119 und 120 Personen ausgewertet. Die mittlere Zeit unter Hypoglykämie änderte sich von 3,38 Stunden/ Tag zu Beginn der Studie auf 2,03 Stunden/Tag nach 6 Monaten (Baseline adjustierte mittlere Veränderung -1,39 Stunden) in der Interventionsgruppe und von 3,44 h/Tag auf 3,27h /Tag in der Kontrollgruppe (-0,14 Stunden), wobei die Differenz zwischen den Gruppen -1,24 Stunden (SE 0,239, p<0,0001) betrug, was einer Verminderung von 38\% der Zeit in Hypoglykämie in der Interventionsgruppe entspricht. Geräteassoziierte Hypoglykämien und unerwünschte Ereignisse wurden nicht berichtet. 13 Unerwünschte Ereignisse wurden bei 10 Teilnehmer im Sinne einer sensorbedingten Allergie berichtet (eine ernste, drei moderate); einmal wurde Juckreiz berichtet (mild), einmal Rötung (moderat) dokumentiert. Es zeigten sich 10 schwere unerwünschte Ereignisse (fünf in jeder Gruppe) von 9 Teilnehmern, die nicht im Zusammenhang mit dem Gerät standen. Die Studie wurde von Abbott finanziell unterstützt. finiert als $<3,9 \mathrm{mmol} / \mathrm{l}[70$ $\mathrm{mg} / \mathrm{dl}]$, im Vergleich Baselinestatus zu 6 Monaten Intervention in der kompletten Studiengruppe. Alle Teilnehmer wurden randomisiert, wobei Schwangere ausgeschlossen wurden.
Schlussfolgerungen: Die Anwendung vom neuartigen FlashGlukose-Monitoring reduzierte die Zeit unter Hypoglykämie bei erwachsenen Patienten mit gut eingestelltem Typ-1-Diabetes. In Zukunft sollten Studien durchgeführt werden, die die Wirksamkeit dieser Technologie sowohl bei schlechter eingestellten als auch jüngeren Typ-1-Diabetes-Patienten testen.

\section{- Kommentar von Prof. Dr. med. Nanette Schloot}

\section{Einfache Messung, positive Stoffwechselwirkung}

Anders als bei Einführung neuer Medikamente wurde das Free Style Libre schon vermarktet, als noch kaum Studienergebnisse publiziert waren. Es ist gut zu wissen, dass die nun geliefert werden und bisher schön zeigen, dass durch das Monitoring bei geschulten Patienten offenbar die Blutzuckerwerte besser werden und weniger Hypoglykämien auftreten. Das künstliche Pankreas (closed loop) wird vermutlich noch bessere Ergebnisse liefern, erst einmal zeigt diese Studie jedoch, wie sich eine vereinfachte, gut durchführbare Messung des Blutzuckers positiv auf die Stoffwechsellage auswirken kann.

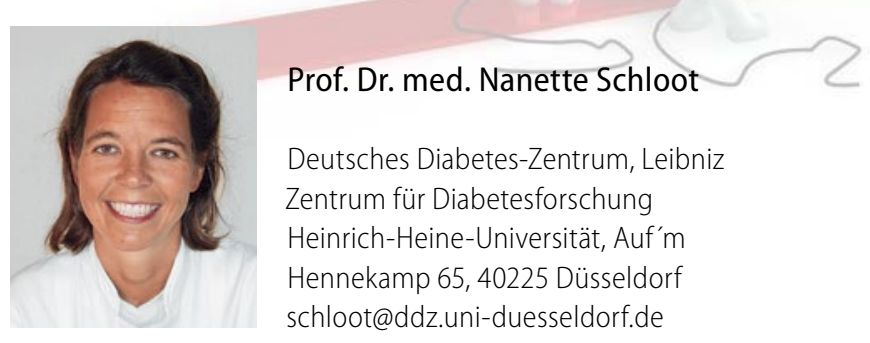

